\title{
Inter-hemispheric asymmetry of nigrostriatal dopaminergic lesion: a possible compensatory mechanism in Parkinson's disease
}

\author{
Javier Blesa ${ }^{1,2}$ *, Carlos Juri ${ }^{1,3}$,Miguel Á. García-Cabezas ${ }^{4,5}$, Rebeca Adánez $^{1,2}$, \\ Miguel Á. Sánchez-González ${ }^{4,5}$, Carmen Cavada ${ }^{4,5}$ and José A. Obeso ${ }^{1,2}$
}

\author{
' Laboratorio de Trastornos del Movimiento, Neurociencias, Centro de Investigación Médica Aplicada, Departamento de Neurología y Neurocirugía, \\ Clínica Universidad de Navarra, Pamplona, Spain \\ ${ }^{2}$ Centro de Investigación Biomédica en Red sobre Enfermedades Neurodegenerativas, Instituto Carlos III, Ministerio de Ciencia e Innovación, Madrid, Spain \\ ${ }^{3}$ Departamento de Neurología, Pontificia Universidad Católica de Chile, Santiago, Chile \\ ${ }^{4}$ Departamento de Anatomía, Histología y Neurociencia, Facultad de Medicina, Universidad Autónoma de Madrid, Madrid, Spain \\ ${ }^{5}$ Instituto de Investigación Sanitaria IdiPAZ, Madrid, Spain
}

\section{Edited by:}

Jose Bargas, Universidad Nacional Autónoma de México, Mexico

Reviewed by:

Alessandro Stefani, University of Rome, Italy

M. Gustavo Murer, Universidad de

Buenos Aires, Argentina

*Correspondence:

Javier Blesa, Centro de Investigación

Médica Aplicada-Neurociencias,

Universidad de Navarra, Pio XII 35,

Pamplona 31008, Spain.

e-mail: jblesa@unav.es
The onset of Parkinson's disease (PD) is characterized by focal motor features in one body part, which are usually correlated with greater dopaminergic depletion in the contralateral posterior putamen. The role of dopamine (DA) hemispheric differences in the onset and progression of motor symptoms of PD, however, remains undefined. Previous studies have demonstrated that unilateral manipulations of one nigrostriatal system affect contralateral DA turnover, indicating a functional and compensatory inter-dependence of the two nigrostriatal systems. In preliminary data obtained by our group from asymmetric PD patients, a higher asymmetry index as measured by 6-[ $\left.{ }^{18} \mathrm{~F}\right]$ fluoro-L-dopa $\left({ }^{18} \mathrm{~F}\right.$-DOPA) positron emission tomography (PET) was associated with a higher threshold (i.e., greater dopaminergic loss) for the onset of motor symptoms in the less-affected side. To further elucidate the underlying basis for this, we carried out a complementary study in monkeys using PET to assess and correlate the degree of dopaminergic striatal depletion with motor activity. Control and 1-methyl-4-phenyl-1,2,3,6-tetrahydropyridine (MPTP)-intoxicated monkeys with symmetrical lesions were characterized behaviorally and with ${ }^{18}$ F-DOPA PET. In parallel, an acute lesion was inflicted in the nigrostriatal projection unilaterally in one monkey, generating a $30 \%$ dopaminergic depletion in the ipsilateral striatum, which was not associated with any noticeable parkinsonian feature or deficit. The monkey remained asymptomatic for several months. Subsequently, this monkey received systemic MPTP, following which motor behavior and PET were repeatedly evaluated during progression of parkinsonian signs. The brains of all monkeys were processed using immunohistochemical methods. Our results suggest that the onset of motor signs is related to and influenced by the dopaminergic status of the less-affected, contralateral striatum. Although this work is still preliminary, the study agrees with our general hypothesis of hemispheric inter-dependence in the compensation of striatal DA deficit in PD.

\section{Keywords: MPTP, Parkinson's disease, PET, dopaminergic, ${ }^{18}$ F-DOPA, compensatory mechanisms}

\section{INTRODUCTION}

Parkinson's disease (PD) is a neurodegenerative disorder characterized mainly by neuronal cell loss predominantly affecting the nigrostriatal dopaminergic pathway (Dickson et al., 2009). However, disease progression is also associated with neuronal loss in the serotonergic, cholinergic, and noradrenergic neurotransmitter systems and with widespread synuclein pathology (Halliday et al., 2008).

The loss of dopaminergic striatal innervation in PD progresses over a period of years before classical motor features arise (Nandhagopal et al., 2009). Accordingly, very powerful compensatory mechanisms must therefore be activated in order to maintain motor activity within normal clinical limits during this pre-symptomatic stage, now recognized to last for a significant period of time (mean 7 years). Indeed, initial motor manifestations typically appear when about $70-80 \%$ of striatal nerve terminals and $50-60 \%$ of substantia nigra pars compacta (SNc) neurons have been lost (Bernheimer et al., 1973; Fearnley and Lees, 1990, 1991). Remarkably, such a large reduction is associated with very mild clinical manifestations confined to a body segment, i.e., slowness of one hand, tremor of the foot, shoulder rigidity, etc., which is paralleled by unequal striatal innervation and asymmetric dopaminergic neuronal loss (Fearnley and Lees, 1991).

One essential feature of PD at onset is the focal and asymmetrical distribution of motor signs, the cause of which remains 
elusive (Djaldetti et al., 2006). Animal models generally produce generalized bilateral deficits (Beal, 2010), which preclude analysis of this essential early asymmetric characteristic. Indeed, the basal ganglia model and its variants that have been developed since the mid-1980s have been unable to address the role and functional significance of inter-hemispheric connectivity. Thus, basal ganglia pathophysiology has been discussed and elaborated without including bilateral, inter-hemispheric connectivity, and possible physiological influences. Accordingly, the role of dopamine (DA) hemispheric differences in the onset and progression of motor features of PD remains by and large undefined (Djaldetti et al., 2006). Previous studies in animal models provoking acute or chronic unilateral lesion of the dopaminergic nigrostriatal system have shown a reciprocal interaction between the intact and DA-depleted striatum (Nieoullon et al., 1977; Andersson et al., 1980; Van Camp et al., 2010). Some previous reports studying the effects of unilateral and bilateral partial lesions of the nigrostriatal system in 6-hydroxydopamine (6-OHDA) rats suggested an interdependent regulation of the two nigrostriatal systems, which could provide some compensatory support for the lesioned hemisphere (Rodriguez et al., 1990; Castellano et al., 1993; Roedter et al., 2001).

In earlier 6- $\left[{ }^{18} \mathrm{~F}\right]$ fluoro-L-dopa $\left({ }^{18} \mathrm{~F}-\mathrm{DOPA}\right)$ positron emission tomography (PET) studies on PD patients, we obtained data which strongly suggest that complete or partial indemnity of striatal DA in one hemisphere increases significantly the capacity of the primarily damaged striatum to compensate for DA depletion (Juri et al., 2009). Here we compared the features of 1-methyl-4-phenyl1,2,3,6-tetrahydropyridine (MPTP)-treated monkeys of different symptom severity with those observed in one monkey with a surgical lesion of the nigrostriatal system followed up with progressive systemic MPTP administration to induce an asymmetrical parkinsonism. This represents a preliminary case-study regarding an asymmetrical model where the onset of motor features and striatal DA depletion has been monitored in vivo by using ${ }^{18} \mathrm{~F}$-DOPA PET. We realize the limitations of an $(n=1)$ observation. Nevertheless, this is a model which mimics more closely the onset and evolution of motor features in PD patients, and it is therefore potentially very useful to ascertain newer therapeutic agents.

\section{MATERIALS AND METHODS \\ ANIMALS}

Seventeen monkeys (Macaca fascicularis; $4.7 \pm 1.1$ years old) from RC Hartelust (Tilburg, The Netherlands), weighing $3.6 \pm 1.02 \mathrm{~kg}$, were included in this study. Animals were housed in an animal room under standard conditions of air exchange (16 l/min), humidity (50\%), and light/dark cycles ( 8 a.m. to 8 p.m.) and were fed fresh fruit and commercial pellets, with free access to water. All procedures were performed according to the European Council Directive 86/609/EEC and in accordance with the Society for Neuroscience Policy on the Use of Animals in Neuroscience Research. The University of Navarra Ethics Committee for Animal Testing and state authorities approved the experimental design.

\section{Bilateral symmetric parkinsonism}

Parkinsonism was induced as previously reported (Blesa et al., 2010) by systemic administration (i.v.) of MPTP using a low-dose regimen $(0.5 \mathrm{mg} / \mathrm{kg}$ every 2 weeks) to obtain partial, slowly progressive and bilateral degeneration of the nigrostriatal dopaminergic system. In order to classify the motor status, a validated motor score (Kurlan scale; Kurlan et al., 1991) was used. Four animals were included in each group and classified as: (1) Control, normal, intact monkeys $(n=4)$; (2) Asymptomatic, animals that received MPTP but never showed any evidence of motor impairment $(n=4)$; (3) Initial Parkinsonian, this group consisted of four monkeys that developed mild motor features for several weeks. Subsequently, without further intoxication they showed minimal residual parkinsonian features or complete motor recovery. Accordingly, they were considered to be at the threshold between the asymptomatic and symptomatic stages; (4) Stable Parkinsonian, were those monkeys that exhibited parkinsonian features after MPTP and remained affected thereafter $(n=4)$. Each animal had to remain stable for at least 1 month in the corresponding motor state to be classified in a given group.

To generate the model, motor behavior was analyzed weekly after MPTP injection. Inter-subject sensitivity to MPTP leads to variable outcomes during the initial intoxication period (first two doses). A monkey that was free of motor manifestations after the second injection was considered to be Asymptomatic and received no further MPTP. All other monkeys developed parkinsonian features after the initial MPTP doses. Four of these animals with initial motor symptoms were left to improve until they exhibited only minimal or no motor (Initial Parkinsonian) features without further intoxication, while others continued to receive MPTP until a stable parkinsonian state was obtained (Stable Parkinsonian). The latter showed bilateral and symmetric motor deficits which persisted unchanged until the end of the study.

\section{Bilateral asymmetric parkinsonism (case-study monkey)}

One monkey ( $M$. fascicularis; 4 years old, weight $2.5 \mathrm{~kg}$ ) was used in this preliminary study. First, a unilateral lesion of the right nigrostriatal pathway was made. Following anesthesia (ketamine/midazolam) the monkey was fixed to a stereotactic device and a trepanus was performed following the right subthalamic nucleus (STN) coordinates $(6 \mathrm{~mm}$ posterior and $4 \mathrm{~mm}$ lateral to anterior commissure and $27.5 \mathrm{~mm}$ ventral to the dura). The STN was localized by neuronal extracellular recordings. A mechanical lesion was made ventromedial to STN without affecting it.

Subsequently, several months after establishing the initial focal lesion which interrupted the nigrostriatal projection unilaterally, the same monkey received systemic administration (i.v.) of MPTP using a low-dose regimen of $0.25 \mathrm{mg} / \mathrm{kg}$ every 2 weeks on four occasions which resulted in early and mild unilateral parkinsonian features contralateral to the previously lesioned side. Then, $0.5 \mathrm{mg} / \mathrm{kg}$ was given every 2 weeks until the development of bilateral motor features consistent with stable parkinsonism. This allowed the severity and evolution of motor features to be separated and for each hemibody to be designated according to the Primary Affected Side (the right side of the body) or Secondary Affected Side (the left side of the body). 


\section{MOTOR BEHAVIOR \\ Peanuts test}

This manual motor task test measured the time taken for the monkey to reach out and retrieve four peanuts that had been placed in front of it. The test was performed with the monkey comfortably seated in a cage specifically prepared for this purpose, with a methacrylate front panel and two symmetrical holes with a diameter of $5 \mathrm{~cm}$. Each hole corresponded to the positioning of each of the hands. Peanuts were placed within circles $(2 \mathrm{~cm}$ in diameter $)$ in a tray directly in line with the panel holes. The examiner recorded the time taken for the monkey to perform the test five times with each hand alternately.

\section{PET STUDIES}

Positron emission tomography studies were conducted using the radioligand ${ }^{18} \mathrm{~F}$-DOPA in a dedicated small animal Philips Mosaic tomograph (Cleveland, OH, USA). The radiotracer was intravenously injected through the saphenous vein simultaneously to the beginning of a list mode study. To block peripheral decarboxylation of ${ }^{18} \mathrm{~F}$-DOPA, $50 \mathrm{mg}$ of carbidopa was given orally $1 \mathrm{~h}$ prior to the scans and anesthesia. Anesthesia was initially induced by intramuscular injections of ketamine $(10 \mathrm{mg} / \mathrm{kg})$ and midazolam $(1 \mathrm{mg} / \mathrm{kg})$ to allow preparation and handling of the monkeys. Anesthesia was maintained during the scans with a mixture of ketamine $(5 \mathrm{mg} / \mathrm{kg})$ and midazolam $(0.5 \mathrm{mg} / \mathrm{kg})$.

Studies were conducted using baseline conditions and after stabilization in the different motor states in the bilaterally lesioned animals. Regarding the case-study monkey with unilateral lesion of the nigrostriatal pathway and subsequent MPTP intoxication, the assessments were conducted following the mechanical lesion, and then 2 weeks after the second MPTP dose when the monkey was still asymptomatic, after the onset of unilateral parkinsonism, and when bilateral parkinsonian features had developed. A stabilization period was also allowed after the onset of unilateral and bilateral parkinsonism before conducting the studies. PET data acquisitions and analyses were performed using the standard protocol described previously by our group (Collantes et al., 2009; Blesa et al., 2010).

\section{HISTOLOGICAL ANALYSIS}

Animals were perfused with saline, followed by $4 \%$ paraformaldehyde in phosphate buffer $(\mathrm{PB}, 0.1 \mathrm{M}, \mathrm{pH} 7.4)$ and a series of PB sucrose solutions (5-20\%). Brains were removed from the skull, cut into $40 \mu \mathrm{m}$ thick coronal sections and stored for immunohistochemical procedures (Cavada et al., 1995). Tyrosine hydroxylase $(\mathrm{TH})$ positive cells in the mesencephalon were counted by stereological analysis. Dopaminergic innervation was studied by immunostaining of $\mathrm{TH}$ in the striatum. All analyses were performed on the right hemisphere.

\section{IMMUNOHISTOCHEMICAL PROCEDURES}

Immunoreactivity for TH was localized using the protocol outlined in Sanchez-Gonzalez et al. (2005). Briefly, sections were incubated with mouse anti-TH antibody (1/1000 dilution: MAB5280 Chemicon International, Temecula, CA, USA), followed by a secondary biotinylated antibody (1/400 dilution: AP160B; 1:400;
Chemicon International, Temecula, CA, USA). Visualization was carried out using Avidin/Biotin complex (ABC) Vectastain Elite ${ }^{\circledR}$ (Vector Labs, Burlingame, CA, USA) and diaminobenzidine (DAB; Sigma).

\section{QUANTIFICATION METHODS}

Estimates of the number of TH-immunoreactive(-ir) neuronal bodies in the mesencephalon were quantified according to stereological principles. Eight coronal sections, regularly spaced at intervals of $1200 \mu \mathrm{m}$, from the caudal edges of the mammillary bodies rostrally to the decussation of the superior cerebellar peduncle caudally, were analyzed for each animal. The stereology system consisted of a Zeiss Axioskop optical microscope (Oberkochen, Germany) equipped with a digital camera (AxioCam HRc, Zeiss, Germany) and StereoInvestigator software (version 8.0, MicroBrightField, Williston, VT, USA). The camera settings were maintained throughout the duration of the experiment. We traced arbitrary landmarks based on external references with the StereoInvestigator software to delineate the SNc (A9) and ventral tegmental area (A10) in each region of interest.

The relative density of TH-ir fibers in the caudate and putamen was quantified by measuring the optical density (OD) of immunostaining using Image J (1.41o, NIH, USA) software. A total of seven rostro-caudal sections for each monkey - three anterior and four posterior to the anatomical references - regularly spaced at intervals of $2400 \mu \mathrm{m}$ were examined.

\section{STATISTICS}

All statistics were performed with SPSS software (version 16.0 software for Windows; SPSS Inc., Chicago, IL, USA). Two-sided $p$ values of less than 0.05 and 0.01 were considered significant.

Differences in continuous and normally distributed variables of ${ }^{18} \mathrm{~F}$-DOPA Ki, numbers of TH-ir cells, and optical densities were analyzed using two-way repeated measures analyses of variance (ANOVA), followed by the Bonferroni post hoc test. Normality was checked by the Kolmogorov-Smirnov test.

\section{RESULTS}

\section{MOTOR BEHAVIOR}

Monkeys that received systemic MPTP could be categorized as Asymptomatic, Initial Parkinsonian, and Stable Parkinsonian at the time of analysis ( 9 weeks after last MPTP dose). The group of monkeys labeled as Initial Parkinsonian developed moderate parkinsonism (Kurlan scale score of $\sim 5$ ) after initial MPTP doses and were subsequently left to stabilize (Kurlan scale $\sim 2$ ). Stable Parkinsonian monkeys developed relatively severe bilateral motor deficits during the intoxication phase (Kurlan scale scores of $\sim 17$ ), but some recovery took place during the stabilization period, so that by the end of the experiment they showed moderate parkinsonian features (Kurlan scale score of $\sim 11$ ).

The case-study monkey showed no motor abnormality initially, as evidenced by the results of the motor scale and peanuts test, despite relatively significant dopaminergic depletion of the right striatum (see below). Subsequently, MPTP administration induced progressive bradykinesia, which manifested on the left 


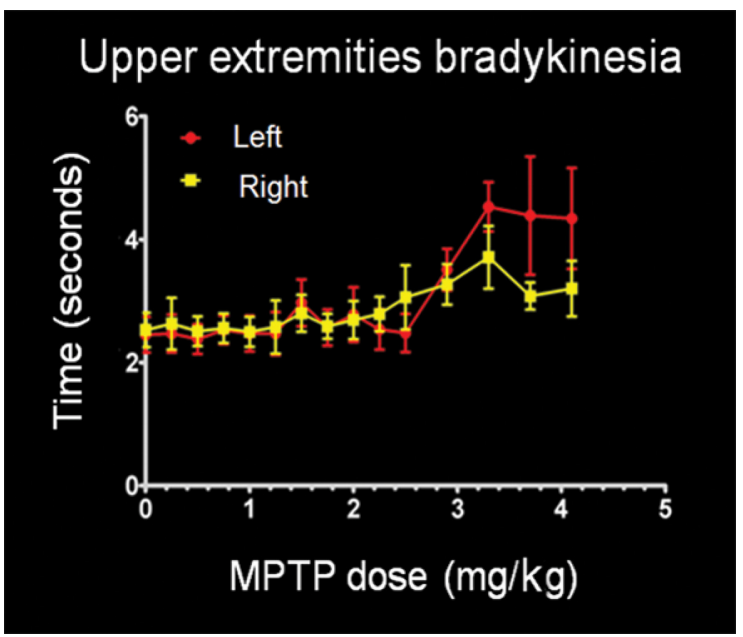

FIGURE 1 | Motor behavior. Evolution of hand bradykinesia using the peanuts test in the case-study monkey with asymmetric involvement of the nigrostriatal dopaminergic system. The left hand (contralateral to the Primary Affected Side) exhibits a progressive increase in time taken to complete the test in parallel with increased 1-methyl-4-phenyl-1,2,3, 6-tetrahydropyridine (MPTP) total dose, while the right hand shows modest involvement

side as clearly shown by the motor scale and the asymmetric loss of motor ability, contralateral to the Primary Affected Side in the peanuts test (Figure 1). As the effect of MPTP progressed, parkinsonian features became more severe and generalized. This monkey thus exhibited bilateral, albeit asymmetrical, parkinsonism by the end of the intoxication period.

\section{PET STUDY}

The ${ }^{18}$ F-DOPA PET showed a linear reduction of uptake in the monkey groups receiving parenteral MPTP (Figure 2). Such a pattern of uptake reduction correlated very significantly with the Kurlan motor score $(p=0.00 ; R=0.77)$, SNc cell loss $(p=0.00$; $R=0.93$ ), and decline of striatal TH immunolabeling $(p=0.00$; $R=0.91)$

In the monkey with the unilateral lesion, ${ }^{18} \mathrm{~F}-\mathrm{DOPA}$ PET uptake was reduced in the Primary Affected Side by $30 \%$ with respect to the contralateral side and continued to decrease bilaterally once MPTP was given. Importantly, the initial asymmetry in ${ }^{18} \mathrm{~F}$-DOPA uptake was maintained during MPTP intoxication until the last stage, when the monkey had developed bilateral motor features (Figure 2). For this one monkey, it was noticeable that the magnitude of dopaminergic depletion associated with the onset of motor manifestations in the lesioned side (Primary Affected Side) was much higher than that observed in the model of symmetric parkinsonisms induced by systemic MPTP administration.

\section{HISTOLOGY}

\section{Tyrosine hydroxylase innervation of the striatum}

For the case-study monkey, TH immunoreactivity reduction was, as expected, greater in the Primary Affected Side (Figure 3). In monkeys with MPTP-induced bilateral deficit, a progressive

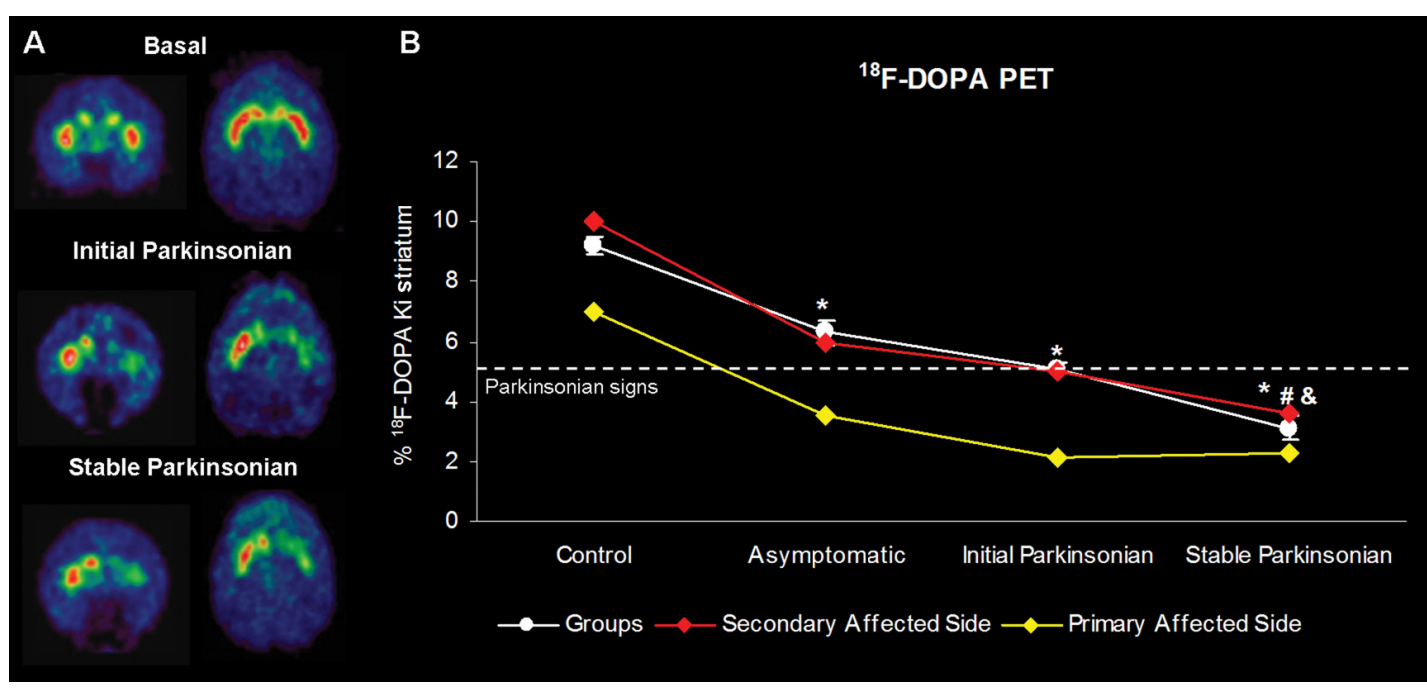

FIGURE 2 | (A) Representative 6-[ $\left.{ }^{18} \mathrm{~F}\right]$ fluoro-L-dopa ( $\left.{ }^{18} \mathrm{~F}-\mathrm{DOPA}\right)$ positron emission tomography (PET) brain images of the asymmetrically affected (case-study) monkey in coronal and transversal orientation at baseline, after 1-methyl-4-phenyl-1,2,3,6-tetrahydropyridine (MPTP) treatment ( Initial Parkinsonian), and after the final MPTP administration ( Stable Parkinsonian). (B) Comparison of 18 F-DOPA uptake in control and MPTP-treated monkeys ( $n=16$; white line), and dopaminergic uptake of the Primary Affected Side (yellow line) and Secondary Affected Side (red line) in the case-study monkey with asymmetric dopamine depletion. In this last-mentioned monkey with the asymmetric lesion, a constant asymmetry between both striata is observed after the unilateral lesion, which remained more or less stable after MPTP administration. Note that the observed depletion at the beginning of parkinsonism in the Primary Affected Side is greater than expected based on the general model. Data represent mean \pm SEM. Symbols indicate significant differences between groups. ${ }^{*} p<0.01$ compared with control; ${ }^{*} p<0.01$ compared with asymptomatic; $\& p<0.05$ compared with recovered group. 


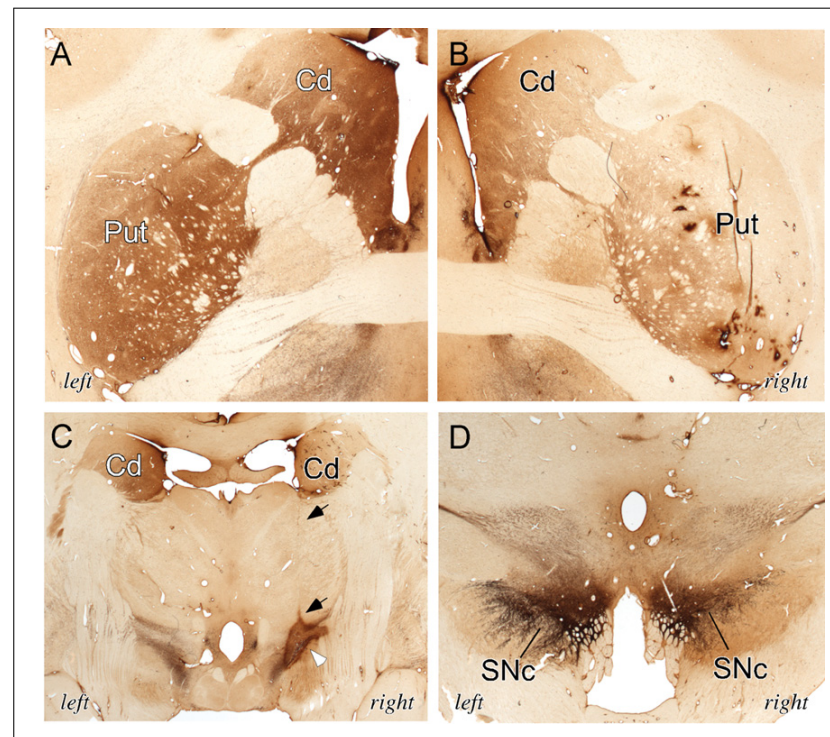

FIGURE 3 | Coronal brain sections showing tyrosine hydroxylase (TH) immunolabeling in the left and right hemispheres of the case-study monkey with a unilateral dopaminergic lesion. Images show the left (A) and right (B) hemispheres at the level of the anterior commissure. Note that the TH immunolabeling is much heavier in the left caudate (Cd) and putamen (Put) than on the right side. (C) Illustration of the track of the electrode (black arrows) that caused the lesion in the nigrostriatal pathway (white arrowhead). Note the asymmetry in TH immunolabeling between the left and right bodies of the caudate nucleus (Cd). (D) TH immunolabeling is weaker on the right versus left substantia nigra pars compacta (SNc). Also note that the subthalamic nucleus appears intact.

reduction in the level of immunoreactivity for $\mathrm{TH}$ was observed in the striatum (Figure 4A), which correlated with the severity of parkinsonism for the different groups. The magnitude of the reduction in the case-study monkey $\mathrm{TH}$ immunoreactivity was close to that (i.e., greatest loss) for the Stable Parkinsonian group (Figure 4A).

\section{Dopaminergic cells in the mesencephalon}

For monkeys receiving MPTP parenterally, the number of TH-ir neurons in A9 declined in parallel with the increased severity of motor deficits (Figure 4B). The minimal cell loss was a 37\% reduction with respect to controls in the Asymptomatic group; this is in comparison to the Stable Parkinsonian group which showed a $75 \%$ reduction. For the case-study monkey, cell counts of TH-ir neuronal cell bodies in the mesencephalon showed bilateral loss of these neurons in the $\mathrm{SNc}$ (A9), which was greater in the Primary Affected Side (Figure 4B) than in the Secondary Affected Side. Moreover, the values fit well with the degree of motor deficit, so that the most severe parkinsonian features in the Primary Affected Side corresponded with a lower cell count than the less severe Secondary Affected Side. Importantly, cell loss in the SNc of the Primary Affected Side was greater even than that of monkeys with Stable Parkinsonism. Cell loss in A10 (ventral tegmental area) was similar to that observed in monkeys with parkinsonism induced by systemic MPTP administration (Figure 4C).

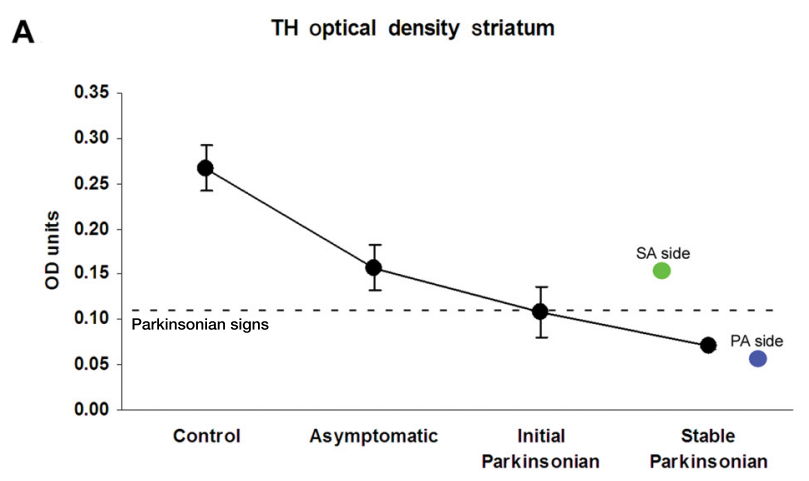

B

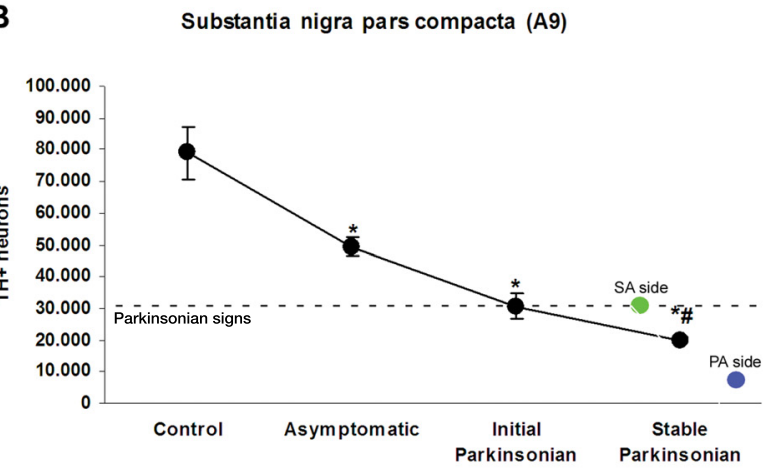

C

Ventral tegmental area (A10)

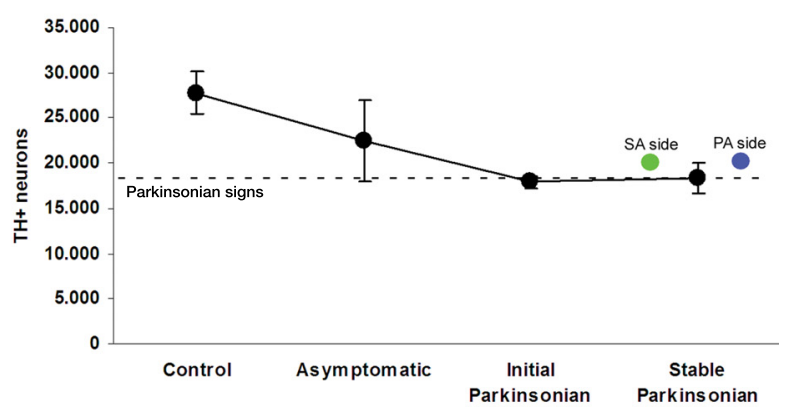

FIGURE 4 | Tyrosine hydroxylase (TH) immunostaining in the striatum and mesencephalon. (A) Line graph showing progressive reduction in the optical density (OD) quantitative analysis of $\mathrm{TH}$ immunostaining in the striatum. Line graphs showing the effect of 1-methyl-4-phenyl-1,2,3, 6-tetrahydropyridine (MPTP) administration on the number of TH-positive cells remaining in the substantia nigra pars compacta (A9) (B), and ventral tegmental area (A10) (C) of control and MPTP-treated monkeys. For comparison, the number of $\mathrm{TH}$-positive cells remaining in each region and the OD in the striatum of the Primary Affected Side (blue circle) and Secondary Affected Side (green circle) of the asymmetric monkey is shown. Data represent mean \pm SEM. Symbols indicate significant differences between groups. ${ }^{*} p<0.01$ compared with control; ${ }^{\#} p<0.01$ compared with asymptomatic.

\section{DISCUSSION}

The manner by which the brain compensates for progressive striatal DA depletion over the course of many years before clinical motor features of PD are manifested is yet to be elucidated. The main recognized compensatory mechanisms include increased dopaminergic pre-synaptic turnover, down-regulation 
of pre-synaptic dopaminergic auto-receptors and DA transporter, and up-regulation of post-synaptic dopaminergic receptors (Hornykiewicz and Kish, 1987; Zigmond et al., 1989; Zigmond, 1997; Brotchie and Fitzer-Attas, 2009). Undoubtedly, the more classic and reputedly compensatory mechanism of striatal depletion in PD consists of an increased DA striatal turnover, as established in the 6-OHDA-lesioned rat model (Zigmond et al., 1990) and in human PD patients (Hornykiewicz, 1975; Zigmond et al., 1990; Pifl and Hornykiewicz, 2006). More recently, some studies have challenged the fundamental role attributed to striatal DA turnover in compensating for PD. Thus, substantia nigra (SN) firing was not found to be significantly increased in the unilateral 6-OHDA rat model (Rodriguez and Gonzalez-Hernandez, 1999), and the changes in striatal DA turnover in the MPTP monkey model were observed too late in the evolution of the process to account for the pre-symptomatic period (Bezard et al., 2001). This led to the suggestion that other compensatory mechanisms beyond the striatum may be more relevant or important in the early phase of PD (Bezard et al., 2003). These may include increased glutamatergic and serotonergic striatal input (Boulet et al., 2008) and modulation of basal ganglia output circuits and beyond (Obeso et al., 2004; Tang et al., 2011).

A possible role of the inter-hemispheric influence of basal ganglia function and compensation of the dopaminergic deficit has been suggested but has received little attention. To this extent, crossed afferents to the striatum from the contralateral $\mathrm{SN}$ and ventral tegmental area were observed in the rat, which persisted after corpus callosotomy (Fass and Butcher, 1981). Nieoullon et al. (1977) showed in cats that infusion of DA in the SNc (resulting in a decreased DA concentration in the striatum) was associated with an increase in the liberation of DA in the contralateral striatum and the opposite effect when the DA infusion was stopped (Nieoullon et al., 1977). In rats with an ibotenic acid-induced striatal lesion, Narang et al. (1993) demonstrated increased striatal D2 receptor mRNA expression contralaterally. Neurophysiologic changes have also been demonstrated contralaterally to unilateral lesions; for example, a decrease in the rate of neuronal discharge in the contralateral STN (Perier et al., 2000) or an increase in the firing rate of contralateral SN GABAergic neurons (GonzalezHernandez et al., 2004). With specific relevance to our current approach, a study comparing the effect of unilateral versus bilateral striatal 6-OHDA lesions in rats showed a marked aggravation of locomotor and exploratory behavior and the use of the forelimbs, suggesting a functional compensatory inter-dependence of the two nigrostriatal systems (Roedter et al., 2001). Finally, we have obtained preliminary findings in PD patients suggesting that the absence of clinically evident signs in early unilateral PD is related not only with the dopaminergic depletion of the less-affected striatum but also to the degree of putamen Ki asymmetry between sides (Juri et al., 2009).

We report here for the first time a direct comparison between symmetrical and asymmetrical dopaminergic depletion in the MPTP monkey model. We readily acknowledge that the results are very preliminary as only one monkey with an asymmetrical lesion has been studied and some un-controlled variables could potentially undermine these preliminary results. For instance, the lesion could have impinged upon the STN or its efferent connections to the globus pallidus pars interna, which would have had an antiparkinsonian effect. However we believe that such a possibility is unlikely anatomically as well as in terms of the putative impact on motor behavior. Thus, the focal lesion was medio-ventral to the STN and the efferent fibers projecting from the motor STN to the motor globus pallidus pars interna and externa are dorso-lateral, and therefore, quite remote from the lesion site. These motor fibers arising from the dorso-lateral STN are well recognized to be responsible for conveying excessive glutamatergic activity, which gives rise to the parkinsonian state. Thus, we have no doubt the motor STN was completely spared in this monkey. In the symmetrical monkeys there was a very powerful correlation between motor behavior and degree of dopaminergic lesion as evaluated by PET in vivo and post-mortem. The case-study monkey with asymmetric lesion did not fit into such a general pattern, showing a higher reduction in ${ }^{18} \mathrm{~F}$-DOPA uptake in the PET studies and greater SNc cell loss than expected for the degree of motor deficit. In other words, the asymmetric monkey tolerated a more severe dopaminergic lesion before becoming fully parkinsonian.

These findings support the hypothesis that the contralateral dopaminergic activity of the less-affected hemisphere plays a role in compensating for dopaminergic depletion of the most affected hemisphere in PD. To our knowledge this is the first concerted attempt to analyze using PET the role of inter-hemispheric asymmetry in DA depletion.

Asymmetry in the onset of the motor manifestations is a characteristic feature of PD. The cause of this asymmetry is not known (Djaldetti et al., 2006), but it could reflect a different vulnerability of the SNc to the factor(s) involved in the genesis of the disorder. Data from PD patients have suggested that those with higher asymmetry could evolve slower than those with a more symmetrical motor involvement (Poewe, 2006), particularly when tremor is the predominant motor manifestation (Djaldetti et al., 2006).

\section{CONCLUSION}

The data presented here indicate that asymmetry of dopaminergic depletion is probably involved in the clinical manifestations of the primarily affected body side in PD patients. The precise mechanisms of such inter-hemispheric influence and inter-dependence are not well understood and warrant further investigation. Our findings open a new experimental approach to study PD and may be highly relevant when considering the early treatment of PD in general, and the application of neuroprotective therapies in particular.

\section{ACKNOWLEDGMENTS}

This work was supported in part by the Plan Nacional de Investigación (SAF2005-08416; SAF2008-04276), Ministerio de Ciencia e Innovación, and by the UTE-CIMA agreement with the Universidad de Navarra. Carlos Juri was supported by the ALBAN Programme, the European Union Programme of High Level Scholarships for Latin America, scholarship No. E07D403507CL. Monkeys were housed and cared for at the CIFA (Centro de Investigación de Farmacología Aplicada) Primate Unit at the University of Navarra. 


\section{REFERENCES}

Andersson, K., Schwarcz, R., and Fuxe, K. (1980). Compensatory bilateral changes in dopamine turnover after striatal kainate lesion. Nature 283, 94-96.

Beal, M. F. (2010). Parkinson's disease: a model dilemma. Nature 466, S8-S10.

Bernheimer, H., Birkmayer, W., Hornykiewicz, O., Jellinger, K., and Seitelberger, F. (1973). Brain dopamine and the syndromes of Parkinson and Huntington. Clinical, morphological and neurochemical correlations. J. Neurol. Sci. 20, 415-455.

Bezard, E., Dovero, S., Prunier, C., Ravenscroft, P., Chalon, S., Guilloteau, D., Crossman, A. R., Bioulac, B., Brotchie, J. M., and Gross, C. E. (2001). Relationship between the appearance of symptoms and the level of nigrostriatal degeneration in a progressive 1-methyl-4-phenyl1,2,3,6-tetrahydropyridine-lesioned macaque model of Parkinson's disease. J. Neurosci. 21, 6853-6861.

Bezard, E., Gross, C. E., and Brotchie, J. M. (2003). Presymptomatic compensation in Parkinson's disease is not dopamine-mediated. Trends $\mathrm{Neu}$ rosci. 26, 215-221.

Blesa, J., Juri, C., Collantes, M., Penuelas, I., Prieto, E., Iglesias, E., MartiCliment, J., Arbizu, J., Zubieta, J. L., Rodriguez-Oroz, M. C., GarciaGarcia, D., Richter, J. A., Cavada, C., and Obeso, J. A. (2010). Progression of dopaminergic depletion in a model of MPTP-induced Parkinsonism in non-human primates. An (18)F-DOPA and (11)C-DTBZ PET study. Neurobiol. Dis. 38, 456-463.

Boulet, S., Mounayar, S., Poupard, A., Bertrand, A., Jan, C., Pessiglione, M., Hirsch, E. C., Feuerstein, C., Francois, C., Feger, J., Savasta, M., and Tremblay, L. (2008). Behavioral recovery in MPTP-treated monkeys: neurochemical mechanisms studied by intrastriatal microdialysis. J. Neurosci. 28, 9575-9584.

Brotchie, J., and Fitzer-Attas, C. (2009). Mechanisms compensating for dopamine loss in early Parkinson disease. Neurology 72, S32-S38.

Castellano, M. A., Rivero, F. L., and Rodriguez, M. (1993). Spontaneous firing of nigrostriatal dopaminergic neurons in split-brain rats. Neurosci. Lett. 162, 1-4.

Cavada, C., Company, T., HernandezGonzalez, A., and Reinoso-Suarez, F. (1995). Acetylcholinesterase histochemistry in the macaque thalamus reveals territories selectively connected to frontal, parietal and temporal association cortices. J. Chem. Neuroanat. 8, 245-257.
Collantes, M., Prieto, E., Penuelas, I., Blesa, J., Juri, C., Marti-Climent, J. M., Quincoces, G., Arbizu, J., Riverol, M., Zubieta, J. L., Rodriguez-Oroz, M. C., Luquin, M. R., Richter, J. A., and Obeso, J. A. (2009). New MRI, 18F-DOPA and 11C-(+)-alphadihydrotetrabenazine templates for Macaca fascicularis neuroimaging: advantages to improve PET quantification. Neuroimage 47, 533-539.

Dickson, D., Braak, H., Duda, J., Duyckaerts, C., Gasser, T., Halliday, G., Hardy, J., Leverenz, J., Del Tredici, K., Wszolek, Z., and Litvan, I. (2009). Neuropathological assessment of Parkinson's disease: refining the diagnostic criteria. Lancet Neurol. 8, 1150-1157.

Djaldetti, R., Ziv, I., and Melamed, E. (2006). The mystery of motor asymmetry in Parkinson's disease. Lancet Neurol. 5, 796-802.

Fass, B., and Butcher, L. L. (1981). Evidence for a crossed nigrostriatal pathway in rats. Neurosci. Lett. 22, 109-113.

Fearnley, J., and Lees, A. (1991). Ageing and Parkinson's disease: substantia nigra regional selectivity. Brain 114(Pt 5), 2283-2301.

Fearnley, J. M., and Lees, A. J. (1990). Striatonigral degeneration. A clinicopathological study. Brain 113(Pt 6), 1823-1842.

Gonzalez-Hernandez, T., BarrosoChinea, P., and Rodriguez, M. (2004). Response of the GABAergic and dopaminergic mesostriatal projections to the lesion of the contralateral dopaminergic mesostriatal pathway in the rat. Mov. Disord. 19, 1029-1042.

Halliday, G., Hely, M., Reid, W., and Morris, J. (2008). The progression of pathology in longitudinally followed patients with Parkinson's disease. Acta Neuropathol. 115, 409-415.

Hornykiewicz, O. (1975). Brain monoamines and parkinsonism. Psychopharmacol. Bull. 11, 34-35.

Hornykiewicz, O., and Kish, S. J. (1987). Biochemical pathophysiology of Parkinson's disease. Adv. Neurol. 45, 19-34.

Juri, C., Arbizu, J., Blesa, J., RodriguezOroz, M. C., Prieto, E., Iglesias, E., Collantes, M., Richter, J. A., Penuelas, I., and Obeso, J. A. (2009). Reciprocal Relationship Between the Striatum Bilaterally and Compensation of Dopaminergic Depletion in Early Parkinson's Disease. An 18F-FDOPA PET Study. Program 52024/J34 2009 Neuroscience Meeting Planner. Chicago, IL: Society for Neuroscience.
Kurlan, R., Kim, M., and Gash, D. (1991). Oral levodopa dose-response study in MPTP-induced hemiparkinsonian monkeys: assessment with a new rating scale for monkey parkinsonism. Mov. Disord. 6, 111-118.

Nandhagopal, R., Kuramoto, L., Schulzer, M., Mak, E., Cragg, J., Lee, C., McKenzie, J., McCormick, S., Samii, A., Troiano, A., Ruth, T., Sossi, V., de la Fuente-Fernandez, R., Calne, D., and Stoessl, A. (2009). Longitudinal progression of sporadic Parkinson's disease: a multi-tracer positron emission tomography study. Brain 132, 2970-2979.

Narang, N., Hunt, M., Pundt, L., Alburges, M., and Wamsley, J. (1993). Unilateral ibotenic acid lesion of the caudate putamen results in D2 receptor alterations on the contralateral side. Exp. Neurol. 121, 40-47.

Nieoullon, A., Cheramy, A., and Glowinski, J. (1977). Interdependence of the nigrostriatal dopaminergic systems on the two sides of the brain in the cat. Science 198, 416-418. Obeso, J. A., Rodriguez-Oroz, M. C., Lanciego, J. L., and Rodriguez Diaz, M. (2004). How does Parkinson's disease begin? The role of compensatory mechanisms. Trends Neurosci. 27, 125-127; author reply 127-128.

Perier, C., Agid, Y., Hirsch, E. C., and Feger, J. (2000). Ipsilateral and contralateral subthalamic activity after unilateral dopaminergic lesion. $\mathrm{Neu}$ roreport 11, 3275-3278.

Pifl, C., and Hornykiewicz, O. (2006). Dopamine turnover is upregulated in the caudate/putamen of asymptomatic MPTP-treated rhesus monkeys. Neurochem. Int. 49, 519-524.

Poewe, W. (2006). The natural history of Parkinson's disease. J. Neurol. 253(Suppl. 7), VII2-VII6.

Rodriguez, M., Castellano, M. A. and Palarea, M. D. (1990). Interhemispheric regulation of dopaminergic ascending systems. Life Sci. 47, 377-384.

Rodriguez, M., and GonzalezHernandez, T. (1999). Electrophysiological and morphological evidence for a GABAergic nigrostriatal pathway. J. Neurosci. 19, 4682-4694.

Roedter, A., Winkler, C., Samii, M., Walter, G. F., Brandis, A., and Nikkhah, G. (2001). Comparison of unilateral and bilateral intrastriatal 6-hydroxydopamine-induced axon terminal lesions: evidence for interhemispheric functional coupling of the two nigrostriatal pathways. J. Comp. Neurol. 432, 217-229.

Sanchez-Gonzalez, M. A., GarciaCabezas, M. A., Rico, B., and Cavada,
C. (2005). The primate thalamus is a key target for brain dopamine. $J$. Neurosci. 25, 6076-6083.

Tang, C. C., Poston, K. L., Dhawan, V., and Eidelberg, D. (2011). Abnormalities in metabolic network activity precede the onset of motor symptoms in Parkinson's disease. J. Neurosci. 30, 1049-1056.

Van Camp, N., Vreys, R., Van Laere, K., Lauwers, E., Beque, D., Verhoye, M., Casteels, C., Verbruggen, A., Debyser, Z., Mortelmans, L., Sijbers, J., Nuyts, J., Baekelandt, V., and Van der Linden, A. (2010). Morphologic and functional changes in the unilateral 6-hydroxydopamine lesion rat model for Parkinson's disease discerned with muSPECT and quantitative MRI. MAGMA 23, 65-75.

Zigmond, M. J. (1997). Do compensatory processes underlie the preclinical phase of neurodegenerative disease? Insights from an animal model of parkinsonism. Neurobiol. Dis. 4, 247-253.

Zigmond, M. J., Abercrombie, E. D., Berger, T. W., Grace, A. A., and Stricker, E. M. (1990). Compensations after lesions of central dopaminergic neurons: some clinical and basic implications. Trends Neurosci. 13, 290-296.

Zigmond, M. J., Berger, T. W., Grace, A. A., and Stricker, E. M. (1989). Compensatory responses to nigrostriatal bundle injury. Studies with 6-hydroxydopamine in an animal model of parkinsonism. Mol. Chem. Neuropathol. 10, 185-200.

Conflict of Interest Statement: The authors declare that the research was conducted in the absence of any commercial or financial relationships that could be construed as a potential conflict of interest.

Received: 21 April 2011; accepted: 25 October 2011; published online: 24 November 2011.

Citation: Blesa J, Juri C, García-Cabezas MÁ, Adánez R, Sánchez-González MÁ, Cavada C and Obeso JA (2011) Interhemispheric asymmetry of nigrostriatal dopaminergic lesion: a possible compensatory mechanism in Parkinson's disease. Front. Syst. Neurosci. 5:92. doi: 10.3389/fnsys.2011.00092

Copyright (C) 2011 Blesa, Juri, GarcíaCabezas, Adánez, Sánchez-González, Cavada and Obeso. This is an open-access article subject to a non-exclusive license between the authors and Frontiers Media $S A$, which permits use, distribution and reproduction in other forums, provided the original authors and source are credited and other Frontiers conditions are complied with. 